\title{
Lepton flavour (universality) violation in rare kaon decays
}

\author{
Lewis C. Tunstall* \\ Albert Einstein Centre for Fundamental Physics, Institute for Theoretical Physics, \\ University of Bern, Sidlerstrasse 5, CH-3012 Bern, Switzerland \\ E-mail: tunstalleitp.unibe.ch
}

\begin{abstract}
Recent results from the LHC's first run have revealed intriguing departures from lepton flavour universality in the semi-leptonic decays of $B$-mesons. We discuss the complementary role that rare kaon decays can provide in testing new physics explanations of these flavour anomalies. In the framework of minimal flavour violation, we relate the chiral low-energy constants involved in $K \rightarrow \pi \ell \ell^{\prime}$ and $K \rightarrow \ell \ell^{\prime}(\ell=\mu$ or $e$ ) with the new physics Wilson coefficients of the $b \rightarrow s$ effective Hamiltonian. We comment on the determination of these low-energy constants at NA62 and future kaon experiments, as well as the required improvements in sensitivity necessary to test the $B$-physics anomalies in the kaon sector.
\end{abstract}

38th International Conference on High Energy Physics 3-10 August 2016

Chicago, USA

${ }^{*}$ Speaker. 


\section{Introduction}

With the completion of Run 1 at the LHC, we find ourselves with several indirect hints that physics beyond the Standard Model (SM) may be lurking in the semi-leptonic decays of $B$-mesons. Among the recent experimental anomalies, two at $\mathrm{LHCb}$ have received considerable attention:

- a $2.6 \sigma$ signal of lepton flavour universality violation (LFUV) in the measured [1] branching fractions of $B \rightarrow K \ell^{+} \ell^{-}$decays $(\ell=\mu$ or $e$ );

- the measured value [2,3] of the angular observable $P_{5}^{\prime}[4]$ in the decay $B \rightarrow K^{*} \mu^{+} \mu^{-}$deviates from the SM prediction at the $2-3 \sigma$ level [5-7].

Taken separately, each deviation is at most a $3 \sigma$ effect; however, when combined with other $b \rightarrow s$ transitions, global fits $[8,9]$ indicate that (a) new physics (NP) is preferred over the SM by $4-5 \sigma$, and (b) the effect is in the $\mu \mu$ modes only. Expressed in terms of the effective Hamiltonian for $b \rightarrow s$ transitions [9]

$$
\mathscr{H}_{\mathrm{eff}}^{|\Delta B|=1}=-\frac{4 G_{F}}{\sqrt{2}} V_{t b} V_{t s}^{*} \sum_{i} C_{i}^{B}(\mu) Q_{i}^{B}(\mu)+\text { h.c. }
$$

the potential NP can be interpreted as contributions to the Wilson coefficients $C_{9,10}^{B}$ of the semileptonic operators

$$
Q_{9}^{B}=\frac{e^{2}}{32 \pi^{2}}\left[\bar{s} \gamma^{\mu}\left(1-\gamma_{5}\right) b\right] \sum_{\ell=e, \mu}\left[\bar{\ell} \gamma_{\mu} \ell\right] \quad \text { and } \quad Q_{10}^{B}=\frac{e^{2}}{32 \pi^{2}}\left[\bar{s} \gamma^{\mu}\left(1-\gamma_{5}\right) b\right] \sum_{\ell=e, \mu}\left[\bar{\ell} \gamma_{\mu} \gamma_{5} \ell\right]
$$

Here we discuss the complementary role that rare kaon decays can provide in testing NP explanations of the $B$-physics anomalies. Our analysis [10] is based on the observation that at low energy scales $\mu \ll m_{t, b, c}$, the strangeness-changing transitions are described in terms of the effective Lagrangian [11]

$$
\mathscr{L}_{\text {eff }}^{|\Delta S|=1}=-\frac{G_{F}}{\sqrt{2}} V_{u d} V_{u s}^{*} \sum_{i} C_{i}(\mu) Q_{i}(\mu)+\text { h.c. },
$$

which contains semi-leptonic operators

$$
Q_{7 V}=\left[\bar{s} \gamma^{\mu}\left(1-\gamma_{5}\right) d\right] \sum_{\ell=e, \mu}\left[\bar{\ell} \gamma_{\mu} \ell\right] \quad \text { and } \quad Q_{7 A}=\left[\bar{s} \gamma^{\mu}\left(1-\gamma_{5}\right) d\right] \sum_{\ell=e, \mu}\left[\bar{\ell} \gamma_{\mu} \gamma_{5} \ell\right]
$$

that are the $s \rightarrow d$ analogues of the $b \rightarrow s$ operators $Q_{9,10}^{B}$ in (1.2). In the framework of minimal flavour violation (MFV), the Wilson coefficients of the two sectors are correlated, and we use this feature to convert knowledge of $C_{7 V, 7 A}$ into bounds on $C_{9,10}^{B}$. The quality of the bounds is hampered by non-perturbative effects from $\mathrm{QCD}$, which are parametrised in terms of the lowenergy constants (LECs) arising in the 3-flavour chiral expansion. In Section 2 we focus on the experimental determination of the LECs involved in $K^{ \pm} \rightarrow \pi^{ \pm} \ell^{+} \ell^{-}$and $K_{L} \rightarrow \ell^{+} \ell^{-}$, and comment on how measurements at NA62 and future kaon experiments may improve the resulting limits on $C_{9,10}^{B}$. Concluding remarks are given in Section 3, and for the analysis of LFUV and LFV in other kaon decays not discussed in this article, we refer the reader to [10]. 


\section{Kaon probes of lepton flavour universality violation}

$2.1 K^{ \pm} \rightarrow \pi^{ \pm} \ell^{+} \ell^{-}$

At low energies, the dominant contribution to $K^{+} \rightarrow \pi^{+} \ell^{+} \ell^{-}$is due to single virtual-photon exchange. The resulting amplitude involves a vector form factor $V_{+}(z)$ which up to $O\left(p^{6}\right)$ in the chiral expansion, can be decomposed in the general form [11]

$$
V_{+}(z)=a_{+}+b_{+} z+V_{+}^{\pi \pi}(z), \quad z=q^{2} / m_{K}^{2} .
$$

Here the LECs $a_{+}$and $b_{+}$parametrise the polynomial part, while the rescattering contribution $V_{+}^{\pi \pi}$ can be determined from fits to $K \rightarrow \pi \pi$ and $K \rightarrow \pi \pi \pi$ data. Chiral symmetry alone does not constrain the values of the LECs, so instead, we consider the differential decay rate $d \Gamma / d z \propto$ $\left|V_{+}(z)\right|^{2}$ as a means to extract $a_{+}$and $b_{+}$from experiment. The resulting fit to the decay spectra from all available high-statistics experiments is given in Table 1.

Now for the crucial point: if lepton flavour universality applies, the coefficients $a_{+}$and $b_{+}$ have to be equal for the $e e$ and $\mu \mu$ channels, which within errors is indeed the case. Since the SM interactions are lepton flavour universal, deviations from zero in differences like $a_{+}^{\mu \mu}-a_{+}^{e e}$ would then be a sign of NP, and the corresponding effect would be necessarily short-distance.

To convert the allowed range on $a_{+}^{\mathrm{NP}}$ into a corresponding range in the Wilson coefficients $C_{7 V}^{\ell \ell}$, we make use of the $O\left(p^{2}\right)$ chiral realization of the $S U(3)_{L}$ current

$$
\bar{s} \gamma^{\mu}\left(1-\gamma_{5}\right) d \leftrightarrow i F_{\pi}^{2}\left(U \partial^{\mu} U^{\dagger}\right)_{23}, \quad U=U(\pi, K, \eta)
$$

to obtain

$$
a_{+}^{\mathrm{NP}}=\frac{2 \pi \sqrt{2}}{\alpha} V_{u d} V_{u s}^{*} C_{7 V}^{\mathrm{NP}} .
$$

Contributions due to NP in $K^{+} \rightarrow \pi^{+} \ell^{+} \ell^{-}$can then be probed by considering the difference between the two channels

$$
C_{7 V}^{\mu \mu}-C_{7 V}^{e e}=\alpha \frac{a_{+}^{\mu \mu}-a_{+}^{e e}}{2 \pi \sqrt{2} V_{u d} V_{u s}^{*}} .
$$

In the framework of MFV, this can be converted into a constraint on the NP contribution to $C_{9}^{B}$ :

$$
C_{9}^{B, \mu \mu}-C_{9}^{B, e e}=-\frac{a_{+}^{\mu \mu}-a_{+}^{e e}}{\sqrt{2} V_{t d} V_{t s}^{*}} \approx-19 \pm 79,
$$

where we have averaged over the two electron experiments listed in Table 1.

\begin{tabular}{cccr}
\hline Channel & $a_{+}$ & $b_{+}$ & Reference \\
\hline$e e$ & $-0.587 \pm 0.010$ & $-0.655 \pm 0.044$ & E865 [12] \\
$e e$ & $-0.578 \pm 0.016$ & $-0.779 \pm 0.066$ & NA48/2 [13] \\
$\mu \mu$ & $-0.575 \pm 0.039$ & $-0.813 \pm 0.145$ & NA48/2 [14] \\
\hline
\end{tabular}

Table 1: Fitted values of coefficients in the vector form factor (2.1). 
Evidently, the determination of $a_{+}^{\mu \mu}-a_{+}^{e e}$ needs to be improved by an $O(10)$ factor in order to probe the parameter space relevant for the $B$-anomalies, whose explanation involves Wilson coefficients $C_{9,10}^{B}=O(1)$ [9]. Improvements of this size may be possible at NA62, especially for the experimentally cleaner dimuon mode which currently has the larger uncertainty.

2.2 $K_{L} \rightarrow \ell^{+} \ell^{-}$

With an eye towards future kaon experiments involving $K_{L}$ beams, we also consider $K_{L} \rightarrow \ell^{+} \ell^{-}$ decays as another potential probe of LFUV. These decays are complementary to $K^{+} \rightarrow \pi^{+} \ell^{+} \ell^{-}$ since they provide the means to constrain NP effects due to axial-vector interactions.

The dominant long-distance contribution is due to $K_{L} \rightarrow \gamma^{*} \gamma^{*} \rightarrow \ell^{+} \ell^{-}$, where the dispersive component of the decay amplitude involves a counterterm $\chi$ that is decomposed in long-and shortdistance parts $\chi(\mu)=\chi_{\gamma \gamma}(\mu)+\chi_{\mathrm{SD}}$. The SM prediction for $\chi_{\mathrm{SD}}$ is known [11], but $\chi_{\gamma \gamma}$ depends on two LECs whose values are not fixed by chiral symmetry. Nevertheless, we invoke the same argument applied to $K^{+} \rightarrow \pi^{+} \ell^{+} \ell^{-}$, and observe that if lepton flavour universality holds, then the SM values of $\chi$ must be equal in both the $e e$ and $\mu \mu$ channels. Then, using the chiral realization (2.2) of the $S U(3)_{L}$ current, one obtains an analogous relation to (2.3) for the NP Wilson coefficient:

$$
C_{7 A}^{\mathrm{NP}}=-\frac{\alpha}{F_{K} G_{F} V_{u d} V_{u s}^{*}}\left(\frac{2 \Gamma_{\gamma \gamma}}{\pi m_{K}^{3}}\right)^{1 / 2} \chi_{\mathrm{NP}}, \quad \Gamma_{\gamma \gamma}=\Gamma\left(K_{L} \rightarrow \gamma \gamma\right) .
$$

The final step is to observe that within the framework of MFV, the difference

$$
C_{7 A}^{\mu \mu}-C_{7 A}^{e e}=-\frac{\alpha}{F_{K} G_{F} V_{u d} V_{u s}^{*}}\left(\frac{2 \Gamma_{\gamma \gamma}}{\pi m_{K}^{3}}\right)^{1 / 2}\left(\chi^{\mu \mu}-\chi^{e e}\right),
$$

is directly related to the Wilson coefficients of the $B$-physics sector:

$$
C_{10}^{B, \mu \mu}-C_{10}^{B, e e}=2.6\left(\frac{3.5 \times 10^{-4}}{V_{t d} V_{t s}^{*}}\right)\left(\chi^{\mu \mu}-\chi^{e e}\right) .
$$

Clearly, the quality of the bounds on $C_{10}^{B, \ell \ell}$ depends on the precision with which $\chi^{\ell \ell}$ can be determined. The present situation is as follows: $\chi$ can be determined (up to a two-fold ambiguity) from the measured $K_{L} \rightarrow \ell^{+} \ell^{-}$rates, with the resulting values shown in Table 2. ${ }^{1}$ Although Solution 2 for the ee channel is easily excluded, the current data are not sufficiently precise to distinguish among the remaining Solutions. Moreover, Solution 1 for the $e e$ channel carries a large uncertainty which needs to be improved in order to test LFUV in the interesting regions of parameter space.

To gain an idea of the improvement in precision required, suppose the uncertainty in $\Gamma\left(K_{L} \rightarrow\right.$ $\ell^{+} \ell^{-}$) could be reduced by a factor of 10 and that the central value remained unchanged. In this idealised scenario, Solution 2 for the $\mu \mu$ channel would be strongly disfavored, given that LFUV (if present at all) should manifest itself as a small effect. Substituting the resulting difference $\chi^{\mu \mu}-\chi^{e e} \sim 1.3 \pm 1.3$ into (2.8) would then yield the bound $C_{10}^{B, \mu \mu}-C_{10}^{B, e e} \approx 3.5 \pm 3.5$. We thus find that the improvement required to obtain competitive bounds on $C_{10}^{B}$ is of similar magnitude to what we found in the analysis of $C_{9}^{B}$ in $K^{+} \rightarrow \pi^{+} \ell^{+} \ell^{-}$.

\footnotetext{
${ }^{1}$ One subtlety involved in the fit is that the equality $\chi^{\mu \mu}=\chi^{e e}$ is assumed to hold to all orders in the chiral expansion. In general $[15,16]$, higher-order corrections involve $m_{\ell}$-dependent terms that produce a shift $\Delta \chi$ which must be subtracted to identify potential LFUV contributions. At the two-loop level, we have estimated [10] the size of the shift to be $\Delta \chi^{\mu \mu}-\Delta \chi^{e e}=-2.8$, in line with explicit calculations based on Canterbury approximants [17].
} 


\begin{tabular}{ccc}
\hline Channel & $\chi$ (Solution 1) & $\chi$ (Solution 2) \\
\hline$e e$ & $5.1_{-10.3}^{+15.4}$ & $-\left(57.5_{-10.3}^{+15.4}\right)$ \\
$\mu \mu$ & $3.75 \pm 0.20$ & $1.52 \pm 0.20$ \\
\hline
\end{tabular}

Table 2: Values of the contact term $\chi\left(M_{\rho}\right)$ extracted from the measured $K_{L} \rightarrow e^{+} e^{-}$and $K_{L} \rightarrow \mu^{+} \mu^{-}$rates.

\section{Remarks and future prospects}

Rare kaon decays offer a potential probe into NP explanations of the flavour anomalies observed by LHCb in semi-leptonic $B$-decays. In the framework of MFV, we have discussed how limits on LFUV and LFV at kaon experiments can be converted into bounds on the Wilson coefficients $C_{9,10}^{B}$ of the $b \rightarrow s$ effective Hamiltonian. In this respect, we have focused on the $K^{+}$decay modes we consider to be of most relevance to the NA62 programme (with $K_{L}$ modes included as a point of comparison):

- $K^{+} \rightarrow \pi^{+} \ell^{+} \ell^{-}$and $K_{L} \rightarrow \ell^{+} \ell^{-}$as a means to constrain LFUV due to vector and axialvector interactions. Although the LECs involved in these modes are poorly constrained from theory, they can be determined via precise experimental measurements. In particular, bounds on the short-distance NP effects can be obtained by considering differences between the ee and $\mu \mu$ parameters. For this method to obtain meaningful bounds on $C_{9,10}^{B}$, we found that order-of-magnitude reductions on the LECs' current uncertainties are required. At NA62, this will require a measurement of the $K^{+} \rightarrow \pi^{+} \ell^{+} \ell^{-}$spectrum which significantly reduces the uncertainties of the NA48/2 data.

We note that in order to convert bounds in the kaon sector into those in $B$-physics, we have worked within the framework of MFV. In general, the potential NP may not satisfy MFV, so from this point of view there are three logical possibilities which can be tested at NA62:

1. if the NP explanations for the $B$-meson anomalies are consistent with MFV, then one should see a signal at the sensitivities discussed in Sections 2;

2. the experimental searches at a sensitivity expected from MFV turn out negative. In this case, one could immediately infer that any NP scenario explaining the $B$-anomalies would require departures from MFV;

3. a signal is observed at current or slightly improved sensitivity. As in point 2 above, we could then rule out NP explanations of the $B$-anomalies based on MFV.

\section{References}

[1] R. Aaij et al. (LHCb), Test of lepton universality using $B^{+} \rightarrow K^{+} \ell^{+} \ell^{-}$decays, Phys. Rev. Lett. 113 (2014) 151601 [arXiv:1406.6482].

[2] R. Aaij et al. (LHCb), Measurement of Form-Factor-Independent Observables in the Decay $B^{0} \rightarrow K^{* 0} \mu^{+} \mu^{-}$, Phys. Rev. Lett. 111 (2013) 191801 [arXiv:1308.1707]. 
[3] The LHCb Collaboration, Angular analysis of the $B^{0} \rightarrow K^{* 0} \mu^{+} \mu^{-}$decay, LHCb-CONF-2015-002, CERN-LHCb-CONF-2015-002.

[4] S. Descotes-Genon, T. Hurth, J. Matias and J. Virto, Optimizing the basis of $B->K^{*}$ ll observables in the full kinematic range, JHEP 1305, 137 (2013) [arXiv:1303.5794].

[5] S. Descotes-Genon, L. Hofer, J. Matias and J. Virto, On the impact of power corrections in the prediction of $B \rightarrow K^{*} \mu^{+} \mu^{-}$observables, JHEP 1412, 125 (2014) [arXiv:1407.8526].

[6] W. Altmannshofer and D. M. Straub, New physics in $b \rightarrow$ s transitions after LHC run 1, Eur. Phys. J. C 75 (2015) 382 [arXiv:1411.3161].

[7] S. Jäger and J. Martin Camalich, Reassessing the discovery potential of the $B \rightarrow K^{*} \ell^{+} \ell^{-}$decays in the large-recoil region: SM challenges and BSM opportunities, Phys. Rev. D 93 (2016) 014028 [arXiv:1412.3183].

[8] W. Altmannshofer and D. M. Straub, Implications of $b \rightarrow s$ measurements, arXiv:1503.06199.

[9] S. Descotes-Genon, L. Hofer, J. Matias and J. Virto, Global analysis of $b \rightarrow$ sll anomalies, JHEP 1606, 092 (2016) [arXiv:1510.04239].

[10] A. Crivellin, G. D’Ambrosio, M. Hoferichter and L. C. Tunstall, Lepton Flavor (Universality) Violation in Rare Kaon Decays, Phys. Rev. D 93 (2016) 074038 [arXiv:1601.00970].

[11] V. Cirigliano, G. Ecker, H. Neufeld, A. Pich and J. Portoles, Kaon Decays in the Standard Model, Rev. Mod. Phys. 84 (2012) 399 [arXiv:1107.6001].

[12] R. Appel et al. [E865 Collaboration], A New measurement of the properties of the rare decay $K^{+} \rightarrow \pi^{+} e^{+} e^{-}$, Phys. Rev. Lett. 83 (1999) 4482 [arXiv:hep-ex/9907045].

[13] J. R. Batley et al. [NA48/2 Collaboration], Precise measurement of the $K^{+} \rightarrow \pi^{+} e^{+} e^{-}$decay, Phys. Lett. B 677 (2009) 246 [arXiv:0903.3130].

[14] J. R. Batley et al. [NA48/2 Collaboration], New measurement of the $K^{+} \rightarrow \pi^{+} \mu^{+} \mu^{-}$decay, Phys. Lett. B 697 (2011) 107 [arXiv:1011.4817].

[15] G. D’Ambrosio, G. Isidori and J. Portoles, Can we extract short distance information from $B\left(K_{L} \rightarrow \mu^{+} \mu^{-}\right)$?, Phys. Lett. B 423 (1998) 385 [arXiv:hep-ph/9708326].

[16] G. Isidori and R. Unterdorfer, On the short distance constraints from $K_{L, S} \rightarrow \mu^{+} \mu^{-}$, JHEP 0401, 009 (2004) [arXiv:hep-ph/0311084].

[17] P. Masjuan and P. Sanchez-Puertas, $\eta$ and $\eta^{\prime}$ decays into lepton pairs, JHEP 1608, 108 (2016) [arXiv:1512.09292].

[18] A. Ceccucci, in Proc. Sci., CD15 (2015) 006 [URL: http: / / inyurl . com/na62limits].

[19] D. Ambrose et al. [BNL Collaboration], New limit on muon and electron lepton number violation from $K_{L}^{0} \rightarrow \mu^{ \pm} e^{\mp}$ decay, Phys. Rev. Lett. 81 (1998) 5734 [arXiv:hep-ex/9811038]. 